\title{
Consistency check of degradation mechanism between natural storage and enhancement test for missile servo system
}

\author{
Wang $X u^{1,2}$ and Sun Quan ${ }^{1,3, *}$ \\ 1. College of Information Systems and Management, National University of Defense Technology, Changsha 410073, China; \\ 2. The Second Academy of China Aerospace Science and Industry Corporation, Beijing 100854, China; \\ 3. Hunan Ginkgo Reliability Technology Institute Limited Corporation, Changsha 410100, China
}

\begin{abstract}
Reliability enhancement testing (RET) is an accelerated testing which hastens the performance degradation process to surface its inherent defects of design and manufacture. It is an important hypothesis that the degradation mechanism of the RET is the same as the one of the normal stress condition. In order to check the consistency of two mechanisms, we conduct two enhancement tests with a missile servo system as an object of the study, and preprocess two sets of test data to establish the accelerated degradation models regarding the temperature change rate that is assumed to be the main applied stress of the servo system during the natural storage. Based on the accelerated degradation models and natural storage profile of the servo system, we provide and demonstrate a procedure to check the consistency of two mechanisms by checking the correlation and difference of two sets of degradation data. The results indicate that the two degradation mechanisms are significantly consistent with each other.
\end{abstract}

Keywords: reliability enhancement testing (RET), degradation model, accelerated equation, consistency check, Pearson correlation coefficient.

DOI: $10.21629 / J S E E .2019 .02 .19$

\section{Introduction}

Reliability enhancement testing (RET) belongs to a sort of development testing, also referred as accelerated stress testing [1], which is conducted in the system development phase under more rigorous stress conditions than operational or storage environment to defect design and manufacturing deficiencies. Such testing can hasten performance degradation of the tested system and generate large amounts of performance degradation data rather than failure data, especially for highly reliable systems. Based on the above degradation data and the analysis of degradation mechanisms, accelerated degradation models can be

\footnotetext{
Manuscript received September 28, 2018.

*Corresponding author.

This work was supported by the Natural Science Foundation of Hunan Province (2018JJ2282).
}

established to describe the relationships between degradation characteristics and applied stresses. Once we obtain the accelerated degradation model, the system reliability under normal stress can be extrapolated by the system reliability under the accelerated situation. This can be called extrapolation in operating conditions [2]. In [3-5], the life or reliability under normal stress level was extrapolated based on accelerated degradation models estimated from various accelerated tests. However, this extrapolating method is based on such a crucial assumption: the degradation mechanism under accelerated stress is the same as the one under the normal stress [2,6-8]. That is to say, it is assumed that there is no new mechanism incorporated into the system due to the accelerated stress. It is the important theory basis for RET. But sometimes the accelerated stresses may change the degradation mechanism so that the lifetime estimated from the accelerated test is unable to reflect the real lifetime [9]. In addition, it is difficult to identify the stress range within that the degradation mechanism is not changed [10]. Generally, the consistency of degradation mechanisms is guaranteed by the design of the accelerated degradation test, i.e., the highest stress level is required to be lower than the destruct limit of the test specimen. However, this practice is just a general rule of thumb. Many reliability related test standardizations also have no requirement to identify the consistency of failure or degradation mechanisms [11]. Therefore, it is necessary for the highly reliable systems to test the consistency of degradation mechanisms under various stress levels.

Currently, the methods of checking the consistency can be classified into two categories $[10,12]$ : one is to identify the consistency of mechanisms and the boundary of stresses before accelerated tests by simulation, experiences and a small amount of pre-exposure test data, and the other is based on accelerated degradation models after accelerated tests. For example, Feng [6] proposed a method by 
measuring the difference of the degradation path based on the Spearman rank correlation coefficient. Wang et al. [10] determined the boundary of the degradation mechanism consistency by simulation. In [12-14], the grey theory was utilized to identify the consistency. Based on the constant principle of the acceleration coefficient [15], Xi et al. [16] presented an identification method of the failure mechanism consistency with taking the Gamma process as a study object. Wang et al. [17] investigated a method based on the degradation model after the accelerated test. Guo et al. [18] provided a method to rapidly identify the consistency at the preliminary stage of the accelerated test. In $[19,20]$, F-statistics and Bartlett-statistics were utilized to identify the consistency for various distributions. However, there is no literature about the consistency check of the degradation mechanism of products in RET. The enhancement test data is an important data source to evaluate or predict the reliability level of the developed product. In order to make the best of the RET data, we establish a methodology to check the degradation mechanism consistency of a missile servo system between RET and natural storage conditions. This methodology contributes to verify the rationality of the RET design.

As the object of this study, the missile servo system is a single-use product with long storage periods in its lifecycle. The storage lifetime is considered as its reliability metric during storage periods. The natural storage temperature varies with the alternations of day-nights and four seasons, and therefore the temperature change rate is regarded as the main stress to influence the storage lifetime of the servo system during storage periods.

To accelerate the degradation process of the servo system, we impose more severe temperature change rate on it by the RET. According to standard principles of experimental design, RETs are usually conducted by applying several accelerated stress levels with simple problems, such as step-temperature, rapid cyclic-temperature, stepvibration and combined stresses [5]. Considering the objective of this research and the main life profile of the servo system, we design and conduct two enhancement tests orderly: a cold step-temperature test and a rapid cyclictemperature test. A certain performance is chosen as the degradation metric which behaves sensitive to stress and possesses significant degradation tendency in RETs. Due to the high cost, only one sample can be provided for testing. The sample goes through two test profiles orderly. The rapid cyclic-temperature test follows the cold steptemperature test.

The stress profiles and degradation curves of these two tests are plotted as shown in Fig. 1. The solid curves represent the temperature stress profiles of RETs, and the dashed represent performance degradation of the tested system. In the cold step-temperature test, as shown in Fig. 1(a), the temperature down-step length is $5^{\circ} \mathrm{C}$ when the temperature varies from $-30^{\circ} \mathrm{C}$ to $-40^{\circ} \mathrm{C}$, and becomes $2^{\circ} \mathrm{C}$ when the temperature falls below $-40^{\circ} \mathrm{C}$. The test is carried on until the temperature reaches $-48^{\circ} \mathrm{C}$. The keeping time of every temperature step is $10 \mathrm{~min}$. Degradations are measured and recorded at the end of every temperature step. Degradations increase when the temperature decreases step by step. There is a remarkable increasing tendency for the performance degradation when the temperature decreases below $-35^{\circ} \mathrm{C}$. In the rapid cyclic-temperature test, as shown in Fig. 1(b), there are five temperature change cycles in which the temperature change rate is $20^{\circ} \mathrm{C} / \mathrm{min}$ in the first and the second cycles, $25^{\circ} \mathrm{C} / \mathrm{min}$ in the third and the fourth cycles, and $30^{\circ} \mathrm{C} / \mathrm{min}$ in the fifth cycle.

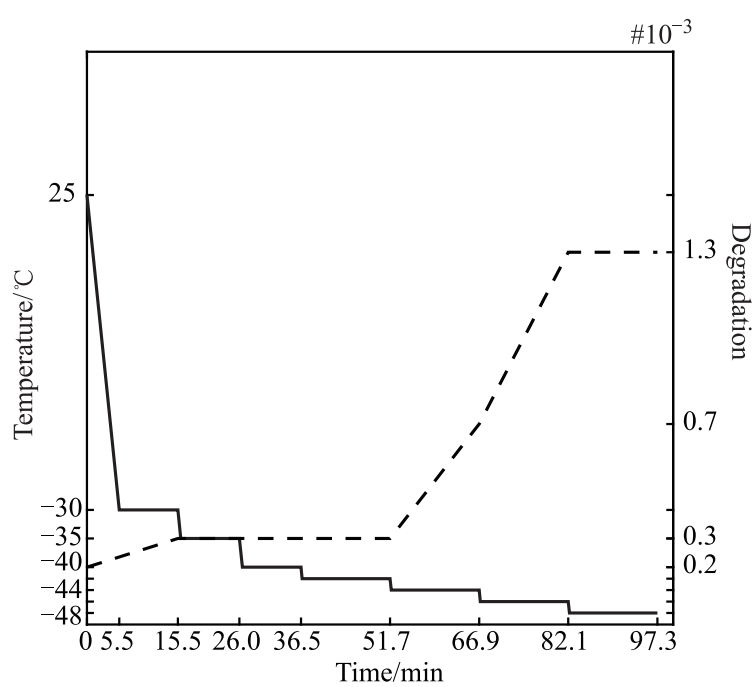

(a) Stress profile and performance degradation curve of the cold step-temperature test

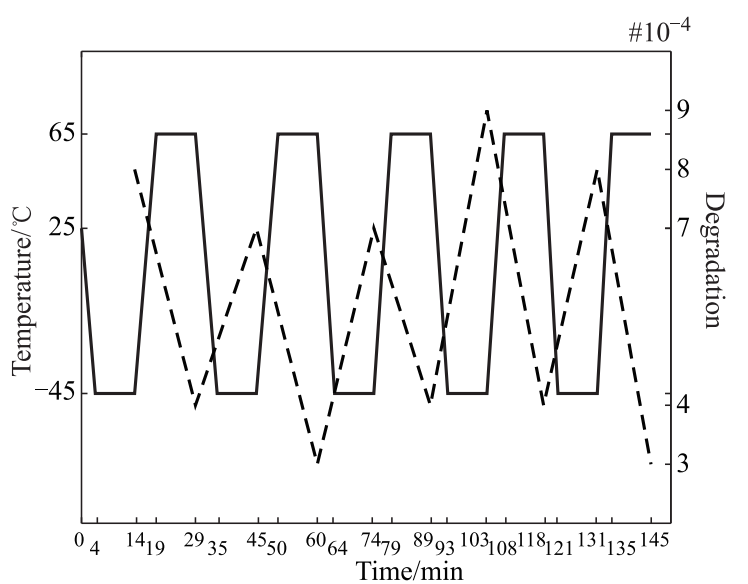

(b) Stress profile and performance degradation curve of the rapid cyclic-temperature test

Fig. 1 Stress profiles and performance degradation curves changing with time 
The temperature change range is from $-45^{\circ} \mathrm{C}$ to $65^{\circ} \mathrm{C}$, and the temperature keeping time is $10 \mathrm{~min}$. The degradations are measured at the end of every temperature keep stage (high and low), and hence there are two measurements in every cycle. Degradations steadily fluctuate in the first three cycles, and the amplitudes of the degradation vibration become large from the fourth cycle. The results indicate that it is necessary to consider the effect of the temperature change rate in the degradation process of the missile servo system.

The research framework of this paper includes three parts as shown in Fig. 2.

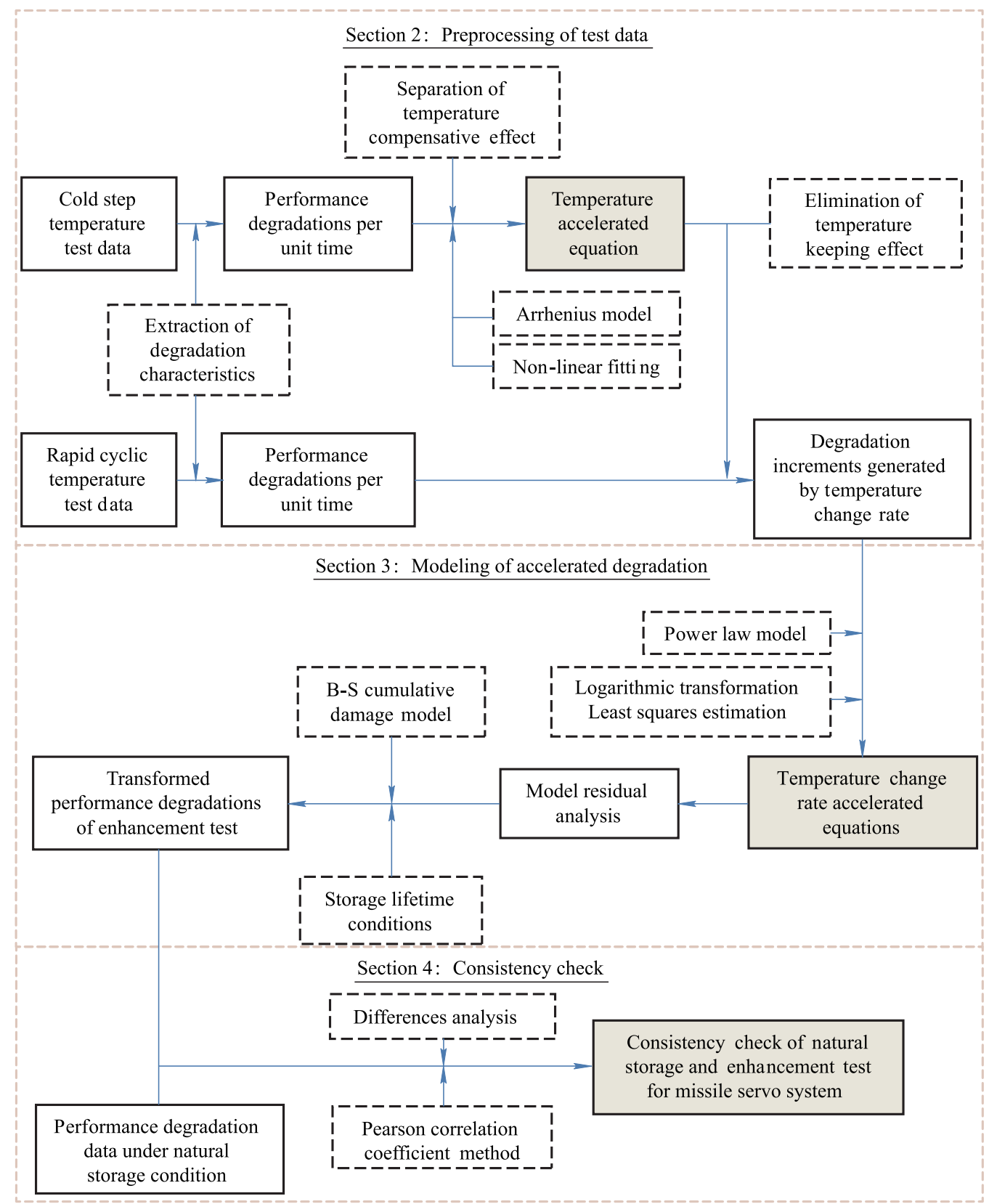

Fig. 2 Research framework of this paper

Section 2 illustrates the preprocessing of the test data. The performance degradations per unit time are obtained from two sets of the test data by using the method of characteristics exaction. Based on the performance degradations per unit time of the cold step temperature test, the temperature accelerated equation can be established by using the method of separating temperature compensative effect, Arrhenius model and non-linear fitting. This equation describes the derived relation between degradation characteristics and temperature. Based on this equation, the per- 
formance degradations of the rapid cyclic temperature test can be transformed into degradation increments generated by the temperature change rate with the method of eliminating the temperature keeping effect.

Section 3 illustrates the modeling of the accelerated degradation caused by the temperature change rate. Based on the degradation increments obtained in Section 2, the temperature change rate accelerated equations can be established by using the power law model, logarithmic transformation and least squares estimation (LSE). For the rapid cyclic temperature test, these equations describe the derived relations between degradation characteristics and the temperature change rate. After residual analysis, the transformed performance degradations from the RET under the natural storage condition can be generated by using the temperature change rate accelerated equations and the B-S cumulative damage model.

Section 4 illustrates the consistency check of the natural storage and RET. We check the consistency of two degradation mechanisms under the natural storage and RET conditions by using the Pearson correlation coefficient method and differences analysis.

\section{Preprocessing of test data}

The observed test data represents the cumulative performance degradation related to the stress profile that the sample has experienced during the RET. In order to establish the degradation accelerated model, we need to preprocess the test data and calculate the performance degradation per unit time at a given stress level.

Let $Y_{i}$ be the individual performance degradation of the sample at stress level $i, q$ is the number of stress levels, then the cumulative degradation after the sample has experienced the $q$ th stress level can be calculated as follows:

$$
W_{q}=\sum_{i=1}^{q} Y_{i}
$$

The total amount of the degradation by time $t$ can be also expressed as follows:

$$
W_{q}(t)=\sum_{i=1}^{q} p_{i} \Delta t_{i}
$$

where $p_{i}$ is an accelerated coefficient of the sample at the stress level $i, \Delta t_{i}$ is the duration of the stress level $i$.

The degradations of the sample at every stress level can be obtained by follows:

$$
\left\{\begin{array}{l}
Y_{1}(t)=W_{1}(t)=p_{1} \Delta t_{1} \\
Y_{2}(t)=W_{2}(t)-W_{1}(t)=p_{2} \Delta t_{2} \\
Y_{q}(t)=W_{q}(t)-W_{q-1}(t)=p_{q} \Delta t_{q}
\end{array} .\right.
$$

Denote the performance degradation per unit time of the sample at the stress level $i$ by $\widetilde{Y}_{i}(t)$, then

$$
\tilde{Y}_{i}=\frac{Y_{i}(t)}{\Delta t_{i}}=p_{i}, \quad i=1,2, \ldots, q .
$$

As shown in Fig. 1(a), eight data points of the degradation are observed in the cold step-temperature test. Then, eight degradation characteristics per unit time can be extracted by (3).

Suppose that the effects of the temperature on the performance degradation are composed of two components: one is restorable, and the other is not restorable. In other words, the restorable component is regarded as a temporary damage which only depends on the temperature of the current stress level, and the remainder is a permanent damage which depends on the stress profile that the sample has accumulatively experienced.

Then, for the cold step-temperature test, the total amount of the degradation of the sample observed at the stress level $i$ can be divided into two components: the temporary damage and the permanent damage.

$$
\left\{\begin{array}{l}
W_{T_{i}}=W_{T_{i}}^{\mathrm{tem}}+W_{T_{i}}^{\mathrm{per}} \\
W_{T_{i}}^{\mathrm{tem}}=\gamma \cdot T_{i} \\
W_{T_{i}}^{\mathrm{per}}=\sum_{j=1}^{i} Y_{j}=\sum_{j=1}^{i} p_{j}^{\text {per }} \Delta t_{j}
\end{array}\right.
$$

where $W_{T_{i}}^{\text {tem }}$ and $W_{T_{i}}^{\text {per }}$ represent the temporary and permanent damage of the sample at the stress level $i$ respectively; $T_{i}$ is the temperature value of the stress level $i ; \gamma$ is an unknown coefficient on the temporary damage which is assumed to depend on the temperature linearly considering the degradation from the temporary damage is insignificant; $p_{j}^{\text {per }}$ is an accelerated coefficient on the permanent damage of the sample at the stress level $i$.

With (2), the degradations of the sample at every stress level can be expressed as follows:

$$
\begin{gathered}
Y_{i}(t)=W_{T_{i}}(t)-W_{T_{i-1}}(t)=\gamma \cdot\left(T_{i}-T_{i-1}\right)+p_{i}^{\text {per }} \Delta t_{i}, \\
i=1,2, \ldots, q .
\end{gathered}
$$

Then, the performance degradations per unit time at every stress level can be rewritten as follows:

$$
\tilde{Y}_{i}=p_{i}^{\text {per }}+\gamma \cdot \frac{\Delta T_{i}}{\Delta t_{i}}, \quad i=1,2, \ldots, q
$$

where $\Delta T_{i}=T_{i}-T_{i-1}$ denotes the temperature increment from the stress level $i-1$ to $i$; therefore, $\frac{\Delta T_{i}}{\Delta t_{i}}$ is the temperature change rate of the stress level $i$.

For the cold step-temperature test, temperature is the main stress resulting in performance degradation. Thus we adopt a typical temperature accelerated model, such as the Arrhenius law which is widely used for the temperatureaccelerated degradation. Then, the relationship between the permanent degradation per unit time $p_{i}^{\text {per }}$ and absolute temperature $T_{i}$ can be proposed as follows: 


$$
p_{i}^{\text {per }}=a \cdot \exp \left(-\frac{b}{T_{i}}\right) .
$$

In the above expression, $a$ is an unknown constant relevant to the material, shape, and machining process of the sample; and $b=\frac{E}{k}, k$ is Boltzmann's constant and $E$ is the activation energy of the reaction.

Substituting (7) into (6) yields the relationships between $\widetilde{Y}_{i j}$ and $T_{i}$ as follows:

$$
\tilde{Y}_{i}=\gamma \cdot \frac{\Delta T_{i}}{\Delta t_{i}}+a \cdot \exp \left(-\frac{b}{T_{i}}\right)+\varepsilon_{i}, \quad i=1,2, \ldots, q
$$

where $\varepsilon_{i}$ denotes the random error of $\widetilde{Y}_{i}$, which follows a normal distribution, i.e., $\varepsilon_{i} \sim N\left(0, \sigma_{\widetilde{Y}_{i}}^{2}\right)$.

Based on the data $\left\{\tilde{Y}_{i}, \frac{1}{T_{i}}, \frac{\Delta T_{i}}{\Delta t_{i}} ; i=1,2, \ldots, q\right\}$, a nonlinear fitting method is proposed to estimate the parameters of (8), i.e., $\widehat{\gamma}, \widehat{a}, \widehat{b}$ and $\widehat{\sigma}_{\widetilde{Y}_{i}}^{2}$. Thus, the general temperature accelerated equation is given by

$$
\tilde{Y}_{i}=\widehat{\gamma} \cdot \frac{\Delta T_{i}}{\Delta t_{i}}+\widehat{a} \cdot \exp \left(-\frac{\widehat{b}}{T_{i}}\right)+\widehat{\varepsilon}_{i}, \quad i=1,2, \ldots, q .
$$

Note that the permanent degradation per unit time at the stress level $i, \widetilde{Y}_{i}^{\text {per }}$, is subject to a normal distribution with

the mean $\widehat{a} \cdot \exp \left(-\frac{\widehat{b}}{T_{i}}\right)$ and variance $\widehat{\sigma}_{\widetilde{Y}_{i}}^{2}$, i.e.,

$$
\widetilde{Y}_{i}^{\text {per }} \sim N\left(\widehat{a} \cdot \exp \left(-\frac{\widehat{b}}{T_{i}}\right), \widehat{\sigma}_{\widetilde{Y}_{i}}^{2}\right) .
$$

The parameter estimations of $\widehat{\gamma}, \widehat{a}, \widehat{b}$ and $\widehat{\sigma}_{\widetilde{Y}_{i}}^{2}$ based on the cold step-temperature test data are listed in Table 1.

Table 1 Parameter estimations of the cold step-temperature test

\begin{tabular}{cccc}
\hline$\widehat{\gamma}$ & $\widehat{a}$ & $\widehat{b}$ & $\sigma_{\widetilde{Y}_{i}}^{2}$ \\
\hline $2.1386 \times 10^{-6}$ & $1.1806 \times 10^{-5}$ & -0.9957 & $2.5054 \times 10^{-10}$ \\
\hline
\end{tabular}

Thus, the temporary degradation under the stress level $T$ is

$$
W_{T}^{\mathrm{tem}}=2.1386 \times 10^{-6} \cdot T
$$

The permanent degradation under the stress level $T$ follows a normal distribution with the mean 1.1806 . $10^{-5} \cdot \exp \left(\frac{0.9957}{273+T}\right) \cdot t$ and variance $1.1806 \cdot 10^{-5}$ $\exp \left(\frac{0.9957}{273+T}\right) \cdot t$, i.e.,

$$
W_{T}^{\mathrm{per}}(t) \sim N\left(1.1806 \times 10^{-5} \cdot \exp \left(\frac{0.9957}{173+T}\right) \cdot t, 2.5054 \times 10^{-10} \cdot t\right) .
$$

For the rapid cyclic-temperature test, its profile can be divided into two categories of stages: temperature keep stages and temperature change stages. It is assumed that the two categories of stages are independent of each other. Considering that the main stress of the missile servo system is the temperature change rate, we need to eliminate the performance degradations generated at high and low temperature keeping stages from the total degradations of every temperature change cycle. With (9), the degradation at temperature keep step $T_{i}$ can be described as follows:

$$
Y_{i}=\widehat{\gamma} \cdot T_{i}+a \cdot \exp \left(-\frac{b}{T_{i}}\right) \cdot \Delta t_{i}^{\mathrm{keep}}
$$

where $\Delta t_{i}^{\text {keep }}$ is the duration of the temperature keep step $T_{i}$. Then, for every cycle of a rapid cyclic-temperature test, the degradation at the high and low temperature keep stages can be written respectively as follows:

$$
\begin{aligned}
Y_{\text {high }} & =\widehat{\gamma} \cdot T_{\text {high }}+\widehat{a} \cdot \exp \left(-\frac{\widehat{b}}{T_{\text {high }}}\right) \cdot \Delta t^{\text {keep }} \\
Y_{\text {low }} & =\widehat{\gamma} \cdot T_{\text {low }}+\widehat{a} \cdot \exp \left(-\frac{\widehat{b}}{T_{\text {low }}}\right) \cdot \Delta t^{\text {keep }}
\end{aligned}
$$

where $\Delta t^{\mathrm{keep}}$ is the duration of the temperature keep stage in the rapid cyclic-temperature test profile; $T_{\text {high }}$ and $T_{\text {low }}$ are the temperature values of high and low temperature keep stages respectively.

According to (13) and (14), for temperature change stages, the performance degradation increment due to the temperature change rate $Y_{v_{i k}}^{\text {change }}$ can be obtained by eliminating the high and low temperature keep effects, namely

$$
Y_{V_{i k}}^{\text {change }}=Y_{V_{i k}}-Y_{\text {high }}-Y_{\text {low }}
$$

where $Y_{v_{i k}}$ is the degradation of the sample observed at the $k$ th measurement of the stress level $i$. For the rapid cyclic-temperature test, when $k$ is an odd number, $Y_{v_{i k}}^{\text {change }}$ represents the degradation at the down-temperature change stage; when $k$ is an even number, $Y_{v_{i k}}^{\text {change }}$ represents the degradation at the up-temperature change stage.

Furthermore, the degradation per unit time $\tilde{Y}_{v_{i k}}^{\text {change }}$ can be obtained as follows:

$$
\tilde{Y}_{V_{i k}}^{\text {change }}=\frac{Y_{V_{i k}}^{\text {change }}}{\Delta t_{V_{i}}^{\text {change }}}
$$

where $\Delta t_{v_{i}}^{\text {change }}$ is the duration of the temperature change stage at the stress level $i$. 
Then, the mean and variance of the performance degradation per unit time at the stress level $i$ are respectively

$$
\begin{gathered}
\widetilde{\mu}_{i}^{\text {change }}=\frac{\sum_{k=1}^{n} \tilde{Y}_{V_{i k}}^{\text {change }}}{n} \\
\left(\widehat{\sigma}_{i}^{\text {change }}\right)^{2}=\frac{\sum_{k=1}^{n}\left(\widetilde{Y}_{V_{i k}}^{\text {change }}-\widetilde{\mu}_{i}^{\text {change }}\right)^{2}}{n-1} .
\end{gathered}
$$

Since the degradation per unit time is uniform under the same stress and not related to the time of measurement, we will substitute $\tilde{Y}_{v_{i}}^{\text {change }}$ for $\widetilde{Y}_{v_{i k}}^{\text {change }}$ in the following discussion.

The initial performance value of the sample at the beginning of the rapid cyclic-temperature test has not been monitored and recorded as shown in Fig. 1(b). Therefore, we remove the degradation data of the first cycle and only keep the degradation data of the remaining cycles.

The mean $\widetilde{\mu}_{i}^{\text {change }}$ and variance $\left(\widetilde{\sigma}_{i}^{\text {change }}\right)^{2}$ of the degradation per unit time at three levels of temperature change rates can be calculated by (17) and (18). The results are listed in Table 2.

Table 2 Means and variances of degradations per unit time due to temperature change rates

\begin{tabular}{ccc}
\hline $\begin{array}{c}\text { Temperature change } \\
\text { rate } /\left({ }^{\circ} \mathrm{C} / \mathrm{min}\right)\end{array}$ & $\widetilde{\mu}_{i}^{\text {change }}$ & $\left(\widetilde{\sigma}_{i}^{\text {change }}\right)^{2}$ \\
\hline 20 & $0.0850 \times 10^{-3}$ & $1.4452 \times 10^{-9}$ \\
25 & $0.1233 \times 10^{-3}$ & $2.0702 \times 10^{-9}$ \\
30 & $0.1548 \times 10^{-3}$ & $4.7916 \times 10^{-9}$ \\
\hline
\end{tabular}

\section{Degradation modeling and parameters estimates}

For the rapid cyclic-temperature test, the degradation per unit time at temperature change stages of the temperature change rate $V_{i}, \widetilde{Y}_{v_{i}}^{\text {change }}$, depends on the temperature change rate $V_{i}$. Thus the mean $\widetilde{\mu}_{i}^{\text {change }}$ and variance $\left(\widetilde{\sigma}_{i}^{\text {change }}\right)^{2}$ of $\tilde{Y}_{v_{i}}^{\text {change }}$ also depend on $V_{i}$. According to acceleration characteristics of the temperature change rate, we choose a power law model as the form of the temperature change rate accelerated equation, i.e.,

$$
\left\{\begin{array}{l}
\widetilde{\mu}_{i}^{\text {change }}=\alpha_{1} \cdot V_{i}^{\beta_{1}} \\
\left(\widehat{\sigma}_{i}^{\text {change }}\right)^{2}=\alpha_{2} \cdot V_{i}^{\beta_{2}}
\end{array}\right.
$$

where $\alpha_{1}, \beta_{1}, \alpha_{2}$ and $\beta_{2}$ are the unknown parameters that need to be estimated.

According to the B-S cumulative damage theory $[21,22]$, the performance degradation $\widetilde{Y}_{v_{i}}^{\text {change }}(t)$ after the

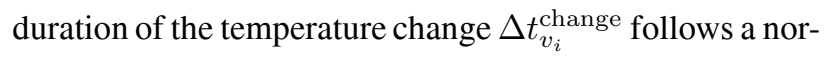
mal distribution with the following mean and variance:

$$
\left\{\begin{array}{l}
\mu_{i}^{\text {change }}(t)=\mathrm{E}\left[Y_{V_{i}}^{\text {change }}(t)\right]=\alpha_{1} \cdot V_{i}^{\beta_{1}} \cdot \Delta t_{V_{i}}^{\text {change }} \\
\left(\sigma_{i}^{\text {change }}\right)^{2}(t)=\operatorname{Var}\left[Y_{V_{i}}^{\text {change }}(t)\right]=\alpha_{2} \cdot V_{i}^{\beta_{2}} \cdot \Delta t_{V_{i}}^{\text {change }}
\end{array}\right.
$$

where $\Delta t_{v_{i}}^{\text {change }}$ is the elapsed time of the temperature change stage at the stress level $V_{i}$ by time $t ; \mu_{i}^{\text {change }}(t)$ and $\left(\sigma_{i}^{\text {change }}\right)^{2}(t)$ are degradation characteristics, referred to as drift coefficient and diffusion coefficient of the degradation process respectively, and $\tilde{Y}_{v_{i}}^{\text {change }}(t)$ is the degradation of the sample that has experienced the stress of the temperature change rate $V_{i}$ for $\Delta t_{v_{i}}^{\text {change }}$ by time $t$.

Take the logarithm on both sides of (19), and we obtain the linear forms as follows:

$$
\left\{\begin{array}{l}
\ln \widetilde{\mu}_{i}^{\text {change }}=\ln \alpha_{1}+\beta_{1} \cdot \ln V_{i} \\
\ln \left(\widehat{\sigma}_{i}^{\text {change }}\right)^{2}=\ln \alpha_{2}+\beta_{2} \cdot \ln V_{i}
\end{array} .\right.
$$

Based on the accelerated test data $\left\{\widetilde{\mu}_{i}^{\text {change }},\left(\widetilde{\sigma}_{i}^{\text {change }}\right)^{2}\right.$, $V_{i}$ \} listed in Table 2 and (21), the estimations $\widehat{\alpha}_{1}, \widehat{\alpha}_{2}, \widehat{\beta}_{1}$ and $\widehat{\beta}_{2}$ can be obtained by the LSE method. The parameter estimations are listed in Table 3.

Table 3 Parameter estimations of the temperature change rate accelerated equations

\begin{tabular}{cccc}
\hline$\widehat{\alpha}_{1}$ & $\widehat{\beta}_{1}$ & $\widehat{\alpha}_{2}$ & $\widehat{\beta}_{2}$ \\
\hline $1.0067 \cdot 10^{-6}$ & 1.4850 & $2.1852 \cdot 10^{-12}$ & 2.9065 \\
\hline
\end{tabular}

Therefore, the temperature change rate accelerated equations for the missile servo system can be written as follows:

$$
\left\{\begin{array}{l}
\mu_{i}^{\text {change }}(t)=1.0067 \cdot 10^{-6} \cdot V^{1.4950} \cdot t \\
\left(\sigma_{i}^{\text {change }}\right)^{2}(t)=1.1852 \cdot 10^{-12} \cdot V^{2.9065} \cdot t
\end{array} .\right.
$$

The fitting curves of the accelerated equations are plotted as shown in Fig. 3. Both of the mean and variance are positively correlated with the temperature change rate.

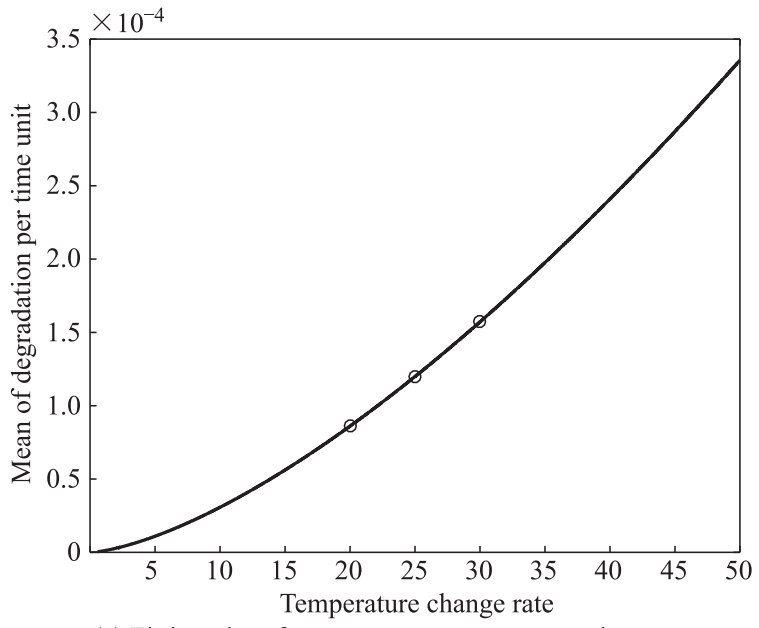

(a) Fitting plot of means versus temperature change rate 


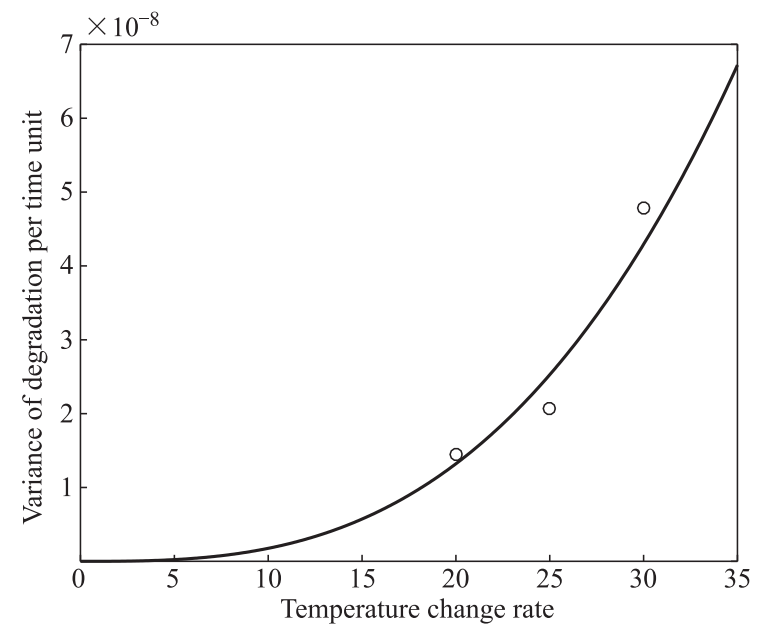

(b) Fitting plot of variance versus temperature change rate

Fig. 3 Fitting plot of the accelerated models in rapid cyclictemperature test

Furthermore, we can verify the above equations by calculating residual errors of $\mu_{i}^{\text {change }}$ and $\left(\widetilde{\sigma}_{i}^{\text {change }}\right)^{2}$. Let $\widehat{\mu}_{\text {mean }}$ and $\widehat{\sigma}_{\text {mean }}^{2}$ be the mean and variance of residual errors of $\mu_{i}^{\text {change }}(t)$ respectively; $\widehat{\mu}_{v a r}$ and $\widehat{\sigma}_{v a r}^{2}$ be the mean and variance of residual errors of $\left(\widetilde{\sigma}_{i}^{\text {change }}\right)^{2}(t)$ respectively. The means and variances of residual errors are listed in Table 4. The results of residual analysis indicate the goodness of fit.

Table 4 Means and variances of residual errors

\begin{tabular}{cccc}
\hline$\widehat{\mu}_{\text {mean }}$ & $\widehat{\sigma}_{\text {mean }}^{2}$ & $\widehat{\mu}_{\text {var }}$ & $\widehat{\sigma}_{\text {var }}^{2}$ \\
\hline $2.5299 \times 10^{-9}$ & $9.2481 \times 10^{-12}$ & $-5.5045 \times 10^{-10}$ & $2.3169 \times 10^{-17}$ \\
\hline
\end{tabular}

\section{Consistency check of natural storage and RET}

In the natural storage profile of the missile servo system, the temperature within 11 months varies within the range of $15-25^{\circ} \mathrm{C}$, and the temperature within one month varies within the range of $5-40^{\circ} \mathrm{C}$. Assume that the temperature at midday is the highest and at midnight is the lowest, and the temperature varies in a circular manner. Therefore, the temperature change rate among 11 months is

$$
\frac{10^{\circ} \mathrm{C}}{12 \mathrm{~h} \times 60 \mathrm{~min}}=0.0139^{\circ} \mathrm{C} / \mathrm{min}
$$

and the temperature change rate among 1 month is

$$
\frac{35^{\circ} \mathrm{C}}{12 \mathrm{~h} \times 60 \mathrm{~min}}=0.0486^{\circ} \mathrm{C} / \mathrm{min} \text {. }
$$

The annual mean of the temperature change rate is

$$
\frac{0.0139 \times 11+0.0496 \times 1}{12}=0.0168^{\circ} \mathrm{C} / \mathrm{min},
$$

i.e., the stress level of the natural storage is $0.0168^{\circ} \mathrm{C} / \mathrm{min}$.
Substitute the temperature change rate $0.0168^{\circ} \mathrm{C} / \mathrm{min}$ into (22) and (23), we can obtain the performance degradation per annual $\widetilde{Y}_{V_{0}}^{\text {year }}$, which follows a normal distribution with mean $\widetilde{\mu}_{0}^{\text {year }}=0.0013$ and variance $\left(\sigma_{0}^{\text {year }}\right)^{2}=$ $1.8535 \cdot 10^{-11}$, namely

$$
\tilde{Y}_{V_{0}}^{\text {year }} \sim N\left(0.0013,1.8535 \times 10^{-11}\right) .
$$

The degradation data of the critical performance parameter under the natural storage condition and the RET are shown in Table 5 and Table 6 respectively. In practice, the missile servo system is tested every half a year and the period of the storage is five years. Thus, the number of measurements is 10 except for the initial state. The temperature change rate of the RET includes three levels of $20^{\circ} \mathrm{C} / \mathrm{min}$, $25^{\circ} \mathrm{C} / \mathrm{min}$ and $30^{\circ} \mathrm{C} / \mathrm{min}$, and ten sets of data are obtained from the enhanment test.

Table 5 Performance degradation data under natural storage condition

\begin{tabular}{cccc}
\hline $\begin{array}{c}\text { Natural } \\
\text { storage } \\
\text { environment }\end{array}$ & Measurements & $\begin{array}{c}\text { Cumulative } \\
\text { test time/min }\end{array}$ & $\begin{array}{c}\text { Critical } \\
\text { performance } \\
\text { degradation }\end{array}$ \\
\hline & 1 & 0 & 0 \\
& 2 & 0.5 & 0.0006 \\
Normal stress & 3 & 1 & 0.0013 \\
$S_{0}$ & 4 & 1.5 & 0.0020 \\
$\left(0.0168^{\circ} \mathrm{C} / \mathrm{min}\right)$ & 5 & 2 & 0.0026 \\
& 7 & 2.5 & 0.0032 \\
& 8 & 3 & 0.0038 \\
& 9 & 3.5 & 0.0044 \\
& 10 & 4 & 0.0052 \\
& 11 & 5.5 & 0.0058 \\
\hline
\end{tabular}

It is well known that the degradation data can be used to characterize the degradation mechanism. Therefore, the consistency check of the degradation mechanism under two stress conditions corresponds to the correlation check and difference analysis of the actual degradation data and the estimated ones based on accelerated models from the RET data. In this paper, we propose a procedure to check the consistency of two degradation mechanisms under the natural storage condition and RET condition. The detailed procedure is recommended as follows:

Step 1 The RET data in Table 6 under three accelerated temperature change rates are preprocessed to obtain the performance degradation characteristics as shown in Table 2 by the method presented in Section 2 .

Step 2 The accelerated degradation equations are estimated by using the degradation characteristics as well as the method presented in Section 3.

With (22) and (23), the temperature change rate accelerated degradation equations are shown as follows:

$$
\left\{\begin{array}{l}
\mu_{i}^{\text {change }}(t)=1.0067 \times 10^{-6} \cdot V^{1.4950} \cdot t \\
\left(\sigma_{i}^{\text {change }}\right)^{2}(t)=1.1852 \times 10^{-12} \cdot V^{2.9065} \cdot t
\end{array} .\right.
$$


Table 6 Performance degradation data under RET condition

\begin{tabular}{|c|c|c|c|c|c|}
\hline $\begin{array}{c}\mathrm{RET} \\
\text { environment } /\left({ }^{\circ} \mathrm{C} / \mathrm{min}\right)\end{array}$ & Measurements & $\begin{array}{l}\text { Cumulative } \\
\text { test time/min }\end{array}$ & $\begin{array}{c}\text { Temperature } \\
\text { change time/min }\end{array}$ & $\begin{array}{c}\text { Temperature } \\
\text { keeping time/min }\end{array}$ & $\begin{array}{l}\text { Critical performance } \\
\text { degradation }\left(10^{-3}\right)\end{array}$ \\
\hline \multirow{8}{*}{20} & & 3.50 & 3.50 & & \\
\hline & 1 & 13.50 & & 10 & 0.8000 \\
\hline & & 19.00 & 5.50 & & \\
\hline & 2 & 29.00 & & 10 & 0.4000 \\
\hline & & 34.50 & 5.50 & & \\
\hline & 3 & 44.50 & & 10 & 0.7000 \\
\hline & & 50.00 & 5.50 & & \\
\hline & 4 & 60.00 & & 10 & 0.3000 \\
\hline \multirow{8}{*}{25} & & 64.40 & 4.40 & & \\
\hline & 5 & 74.40 & & 10 & 0.7000 \\
\hline & & 78.80 & 4.40 & & \\
\hline & 6 & 88.80 & & 10 & 0.4000 \\
\hline & & 93.20 & 4.40 & & \\
\hline & 7 & 103.20 & & 10 & 0.9000 \\
\hline & & 107.60 & 4.40 & & \\
\hline & 8 & 117.60 & & 10 & 0.4000 \\
\hline \multirow{4}{*}{30} & & 121.27 & 3.67 & & \\
\hline & 9 & 131.27 & & 10 & 0.8000 \\
\hline & & 134.93 & 3.67 & & \\
\hline & 10 & 144.93 & & 10 & 0.3000 \\
\hline
\end{tabular}

Step 3 According to the natural storage profile of the servo system, the mean and variance of the performance degradation per half a year can be obtained by the above accelerated equations. With the B-S cumulative damage theory, $\widetilde{Y}_{V_{0}}^{\text {halfyear }}$ follows a normal distribution, i.e., $\widetilde{Y}_{V_{0}}^{\text {halfyear }} \sim N\left(\widetilde{\mu}_{0}^{\text {halfyear }},\left(\widetilde{\sigma}_{0}^{\text {halfyear }}\right)^{2}\right)$. The degradation per half a year can be simulated based on the distribution of $\widetilde{Y}_{V_{0}}^{\text {halfyear }}$. The cumulative degradation estimations $\left\{\widehat{W}_{01}, \widehat{W}_{02}, \ldots, \widehat{W}_{0 n_{0}}\right\}$ at some given time $\left\{t_{01}, t_{02}, \ldots, t_{0 n_{0}}\right\}$ under natural storage condition can be obtained by accumulating $\widetilde{Y}_{V_{0}}^{\text {halfyear }}$.

By substituting the natural storage stress $0.0168^{\circ} \mathrm{C} / \mathrm{min}$ and the time of half a year (i.e., $0.5 \cdot 365 \cdot 24 \cdot 60=$ $3.285 \cdot 10^{5} \mathrm{~min}$ ) into (22) and (23), the mean and variance of performance degradation per half a year are obtained:

$$
\begin{gathered}
\widetilde{\mu}_{0}^{\text {halfyear }}=0.00065 \\
\left(\widetilde{\sigma}_{0}^{\text {halfyear }}\right)^{2}=9.2673 \times 10^{-12} .
\end{gathered}
$$

Then, $\widetilde{Y}_{V_{0}}^{\text {halfyear }} \sim N\left(0.00065,9.2673 \cdot 10^{-12}\right)$.

Subsequently, the degradation per half a year under natural storage condition can be obtained by simulation. By accumulating them, we obtain the cumulative degradation estimations $\left\{\widehat{W}_{01}, \widehat{W}_{02}, \ldots, \widehat{W}_{0 n_{0}}\right\}$ at some given time $\{0.5 a, 1 a, 1.5 a, \ldots, 5 a\}$ as shown in Table 7 .

Step 4 Compare the above estimated data $\left\{\widehat{W}_{01}\right.$, $\left.\widehat{W}_{02}, \ldots, \widehat{W}_{0 n_{0}}\right\}$ with the actual degradation data under the natural storage $\left\{W_{01}, W_{02}, \ldots, W_{0 n_{0}}\right\}$, and calculate the Pearson correlation coefficient of two sets of data:

$$
\widehat{\rho}=\frac{\sum_{j=1}^{n_{0}}\left(W_{0 j}-\overline{W_{0}}\right)\left(\widehat{W}_{0 j}-\overline{\widehat{W}_{0}}\right)}{\sqrt{\sum_{j=1}^{n_{0}}\left(W_{0 j}-\overline{W_{0 j}}\right)^{2} \sum_{j=1}^{n_{0}}\left(\widehat{W}_{0 j}-\overline{\widehat{W}_{0}}\right)^{2}}}
$$

where $\bar{W}_{0}=\frac{1}{n_{0}} \sum_{j=1}^{n_{0}} W_{0 j}, \overline{\widehat{W}}_{0} \frac{1}{n_{0}} \sum_{j=1}^{n_{0}} \widehat{W}_{0 j}$.

Table 7 Estimated results of degradation from RET to natural storage

\begin{tabular}{cccc}
\hline $\begin{array}{c}\text { Natural } \\
\text { storage } \\
\text { Environment }\end{array}$ & Measurements & $\begin{array}{c}\text { Cumulative } \\
\text { test time/min }\end{array}$ & $\begin{array}{c}\text { Transformed } \\
\text { critical } \\
\text { performance } \\
\text { degradation }\end{array}$ \\
\hline & 1 & 0 & 0 \\
& 2 & 0.5 & 0.0007 \\
Normal stress & 3 & 1 & 0.0013 \\
$S_{0}$ & 4 & 1.5 & 0.0020 \\
$\left(0.0168^{\circ} \mathrm{C} / \mathrm{min}\right)$ & 5 & 2 & 0.0026 \\
& 7 & 2.5 & 0.0033 \\
& 8 & 3 & 0.0040 \\
& 9 & 3.5 & 0.0046 \\
& 10 & 4 & 0.0053 \\
& 11 & 4.5 & 0.0060 \\
& 5 & 5 & 0.0066 \\
\hline
\end{tabular}

According to two sets of data from Table 5 and Table 7, the Pearson correlation coefficients during period of 3 years and 5 years $\widehat{\rho}_{3}=0.9992$ and $\widehat{\rho}_{5}=0.9996$. 
Step 5 Determine the correlation criteria. If $\widehat{\rho} \geqslant 0.7$, then two sets of data are strongly correlated, which implies that to some extent the degradation mechanisms of the natural storage and RET are significantly correlated. If $\widehat{\rho} \leqslant 0.4$, then two sets of data have no apparent linear relationship, which means that two mechanisms are not significantly correlated with each other and some new mechanisms may be interpolated due to the accelerated stress; hereby we need to review accelerated stress conditions of the RET. If $0.4 \leqslant \widehat{\rho} \leqslant 0.7$, we are unable to draw final conclusions immediately and need to apply other methods to make further determination.

According to the correlation criterion, both of $\widehat{\rho}_{3}$ and $\widehat{\rho}_{5}$ are greater than 0.7 , then there are strong correlations between two degradation mechanisms for the missile servo system.

Step 6 If $\hat{\rho} \geqslant 0.7$, then we need to further compare means and variances of two sets of data respectively, and calculate the absolute differences of two metrics, namely

$$
\left\{\begin{array}{l}
\Delta \mu=\left|\mu_{\text {estimated }}-\mu_{\text {actual }}\right| \\
\Delta \sigma^{2}=\left|\sigma_{\text {estimated }}^{2}-\sigma_{\text {actual }}^{2}\right|
\end{array}\right.
$$

where $\mu_{\text {estimated }}, \sigma_{\text {estimated }}^{2}$ are the mean and variance of the estimated data; $\mu_{\text {actual }}, \sigma_{\text {actual }}^{2}$ are the mean and variance of the observed data under the natural storage condition. If both of the above differences close to zero or smaller than some given threshold values, it indicates there are no significant difference between two sets of data.

Since the correlation coefficient is greater than 0.7 , we calculate absolute differences of means and variances of two sets of data from Table 5 and Table 7, then $\Delta \mu=$ 0.0004 and $\Delta \sigma^{2}=1.8667 \cdot 10^{-7}$. Two differences approximate zero, and therefore we can draw a conclusion that two degradation mechanisms are consistent with each other.

The preceding procedure provides a way to validate the effectiveness of accelerated equations and the consistency check of various degradation mechanisms.

\section{Conclusions}

In this paper, we utilize the degradation data obtained from the rapid cyclic-temperature test as well as the steptemperature test to develop the performance degradation models based on the theory of probabilistic physics, and apply a method based on Pearson correlation coefficient and differences analysis to check the consistency of degradation mechanisms of the missile servo system under the natural storage condition and the RET condition. Specifically, this paper provides the following conclusions:

(i) This paper proposes a method to extract the degradation characteristics and model the degradation process based on the cumulative damage theory of B-S distribution.

(ii) This paper develops a procedure to establish the temperature change rate accelerated models based on the rapid cyclic-temperature test data and the cold step-temperature test data.

(iii) This paper presents a procedure to check the consistency of degradation mechanisms under two stress conditions.

\section{References}

[1] JIANG P, CHEN X, ZHANG C H. Reliability enhancement testing. Structure \& Environment Engineering, 2003, 30(1): 58-64. (in Chinese)

[2] NELSON W. Accelerated testing: statistical methods, test plans, and data analysis. New York: Wiley, 1990.

[3] SHANG G Z, FU G C, WAN B. Component storage life prediction based on accelerated performance degradation. Electronic Product Reliability and Environment Testing, 2009, 27(5): 32 - 36. (in Chinese)

[4] GAO L N, ZHAO L. Life prediction of electronic equipment based on step-stress accelerated degradation test under temperature stress. Electronic Components and Materials, 2014, 33(6): $72-76$. (in Chinese)

[5] LIAO H T, ELSAYED A. Reliability inference for field conditions from accelerated degradation testing. Wiley Inter Science, 2006, 18(3): 576-587.

[6] FENG J. Consistent test of accelerated storage degradation failure mechanism based on rank correlation coefficient. Journal of Aerospace Power, 2011, 26(11): 2439-2444. (in Chinese)

[7] MEEKER W Q, ESCOBAR L A, LU C J. Accelerated degradation tests: modeling and analysis. Technometrics, 1998, 40: 89-99.

[8] CAREY M B, KOENIG R H. Reliability assessment based on accelerated degradation: a case study. IEEE Trans. on Reliability, 1991, 40: 499-506.

[9] HU J M, BARKER D, DASGUPTA A, et al. Role of failure mechanism identification in accelerated testing. Institute of Environmental Sciences \& Technology, 1993, 36(4): 39-45.

[10] WANG Q C, CHEN Y X, DENG F L, et al. Approach of determining accelerated degradation mechanism consistency's boundary for accelerometers. Journal of Beijing University of Aeronautics and Astronautics, 2012, 38(11): 1512 -1516. (in Chinese)

[11] GUO C S, XIE X S, MA W D, et al. A failure mechanism identification method in accelerated testing. Chinese Journal of Semiconductors, 2006, 27(3): 560-563. (in Chinese)

[12] YAO J,WANG H, SU Q. Consistency identification method of failure mechanism based on grey theory. Journal of Beijing University of Aeronautics and Astronautics, 2013, 39(6): 734 - 738. (in Chinese)

[13] PAN X Q, KANG R. Identification method of failure mechanism consistency for accelerated testing based on grey forecasting. Journal of Beijing University of Aeronautics and Astronautics, 2013, 39(6): 787-791. (in Chinese)

[14] LI X G, WANG Y H. Identification method of failure mechanism consistency by non-equidistance grey theory model. Journal of Beijing University of Aeronautics and Astronautics, 2014, 40(7): 899-904. (in Chinese)

[15] ZHOU Y Q, WENG Z X, YE X T. Study on accelerated factor and condition for constant failure mechanism (I): the case 
for life-time is a random variable. Systems Engineering and Electronics, 1996, 18(1): 55-66. (in Chinese)

[16] XI W J, WANG H W, WANG R. Failure mechanism consistency identification based on acceleration coefficient principle. Journal of Beijing University of Aeronautics and Astronautics, 2015, 41(12): 2198 - 2204. (in Chinese)

[17] WANG H W, XU T X, WANG W Y. Test method of failure mechanism consistency based on degradation model. Journal of Aeronautics, 2015, 36(3): 889-897. (in Chinese)

[18] GUO C S, WANG N, MA W D, et al. Rapid identification of the consistency of failure mechanism for constant temperature stress accelerated testing. Chinese Journal of Physics, 2013, 62(6): 470-474. (in Chinese)

[19] LIN F C, WANG Q C, CHEN Y X, et al. Pseudo-life-based test method of mechanism consistency boundary for accelerated degradation testing. Journal of Beijing University of Aeronautics and Astronautics, 2012, 38(2): 233-238. (in Chinese)

[20] ZHOU Y Q, WENG C X. Statistical inferences of environmental factors for the lognormal distribution. System Engineering and Electronics, 1996, 18(10): 73 -81. (in Chinese)

[21] BIRNBAUM Z, SAUNDERS S. A new family of life distribution. Applied Probability, 1969: 319-327.

[22] ZHAO J Y, SUN Q, PENG B H. Inferences for the BS life model from accelerated degradation tests. Electronic Product
Reliability and Environmental Testing, 2006, 24(1): 11-14. (in Chinese)

\section{Biographies}

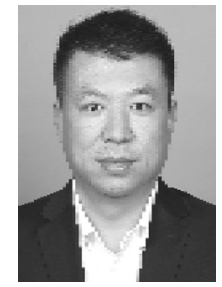

WANG Xu was born in 1979. He is a deputy commander in chief and a chief quality engineer in the Second Academy of China Aerospace Science and Industry Corporation, and a Ph.D. in National University of Defense Technology. His research interests are project quality management and reliability engineering management.

E-mail: wangxu1029@sina.com

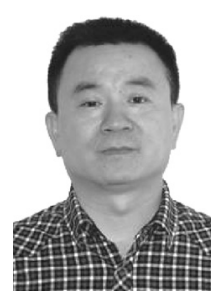

SUN Quan was born in 1973. He is a professor and a Ph.D. supervisor in National University of Defense Technology, and a general manager in Hunan Ginkgo Reliability Technology Institute Limited Corporation. His research interests are lifetime and reliability predication technologies of long-time products.

E-mail: 2183163572@qq.com 